\title{
List of Participants
}

Greg Aldering, University of Minnesota, USA

James Annis, Experimental Astrophysics Group, Fermilab, USA

Kentaro Aoki, National Astronomical Observatory of Japan, Japan

Brent A. Archinal, U.S. Naval Observatory, USA

Robert W. Argyle, Royal Greenwich Observatory, England

Boris Artamonov, Sternberg Astronomical Institute, Russia

Norbert Bade, Hamburger Sternwarte, Germany

Neta Bahcall, Princeton University, USA

Sandro Bardelli, Osservatorio Astronomico di Trieste, Italy

James Bartlett, Observatoire Astronomique de Strasbourg, France

David J. Batuski, University of Maine, USA

Robert Becker, Lawrence Livermore National Laboratory, USA

Charles Beichman, Caltech/JPL, USA

Olivier Bienayme, Observatoire Astronomique de Strasbourg, France

Francois Bonnarel, Observatoire Astronomique de Strasbourg, France

Kirk Borne, Hughes STX - Goddard Space Flight Center, USA

Francois Boulanger, Institut d'Astrophysique Spatiale, France

Wolfgang Brinkmann, MPI für Extraterrestrische Physik, Germany

Noah Brosch, Wise Observatory, Israel

Robert J. Brunner Johns Hopkins University, USA

Blaise Canzian, USRA/U.S. Naval Observatory, USA

Daniela Carollo, Osservatorio Astronomica di Torino, Italy

Jian-sheng Chen, Beijing Astronomical Observatory, China

Cynthia Cheung, NASA/GSFC, USA

Yaoquan Chu, University of Science and Technology, China

Angie Clarke, Space Telescope Science Institute, USA

James Condon, National Radio Astronomy Observatory, USA

Andrew Connolly, Johns Hopkins University, USA

Kem Cook, Lawrence Livermore National Laboratory, USA

Eric Copet, Observatoire de Paris, France

Thomas E. Corbin, US Naval Observatory, USA

Chris Cornuelle, University of Minnesota, USA

Reinaldo de Carvalho, California Institute of Technology, USA

Brian Dennison, Physics Dept., Virginia Tech, USA

Eric Deul, Sterrewacht Leiden, Netherlands

Mark Dickinson, Space Telescope Science Institute, USA

Seth Digel, Hughes STX, NASA/GSFC, USA

Michael Disney, College of Cardiff, University of Wales, Wales

S. George Djorgovski California Institute of Technology, USA

Jesse Doggett, Space Telescope Science Institute, USA 
Daniel Egret, CDS, Observatoire de Strasbourg, France

Nicolas Epchtein, Observatoire de Paris, France

Giovanni G. Fazio, Harvard-Smithsonian Astrophysical Observatory, USA

Eric Feigelson, Penn State University, USA

Paul Feldman, Johns Hopkins University, USA

Holland Ford, Johns Hopkins University, USA

Wolfram Freudling, Space Telescope - ECF, Germany

Ernst F. G. Fürst, Max-Planck-Institut für Radioastronomie, Germany

Yasuo Fukui, Dept. of Physics and Astrophysics, Nagoya Univ., Japan

Roy Gal, California Institute of Technology, USA

Lorraine Garcia, Space Telescope Science Institute, USA

Jonathan P. Gardner, University of Durham, England

Stephen Gauss, U.S. Naval Observatory, USA

Neil Gehrels, NASA/GSFC, USA

Marvin E. Germain, U.S. Naval Observatory, USA

Daniel Golombek, Space Telescope Science Institute, USA

Gretchen Greene, Space Telescope Science Institute, USA

Loretta Gregorini, Istituto di Radioastronomia, CNR, Italy

Herbert Gursky, Naval Research Laboratory, USA

Martin Harwit, USA

Guenther Hasinger, Astrophysikalisches Institut Postsdam, Germany

Michael Hauser, Space Telescope Science Institute, USA

George Hawkins, Space Telescope Science Institute, USA

Jeffrey J.E. Hayes, Space Telescope Science Institute, USA

David Helfand, Columbia University, USA

George Helou, NED/IPAC, USA

Richard C. Henry, Johns Hopkins University, USA

Martin Hiesgen, Observatoire Astronomique de Strasbourg, France

Robert Hindsley, U.S. Naval Observatory, USA

Erik Hoeg, Copenhagen University Observatory, Denmark

Ulrich Hopp, Universitaetssternwarte Munich, Germany

Don Horner, Goddard Space Flight Center, USA

Jiasheng Huang, Univ of Hawaii - Institute for Astronomy, USA

Takashi Ichikawa, Kiso Observatory, University of Tokyo, Japan

Helmut Jenkner, Space Telescope Science Institute, USA

Justin Jonas, Rhodes University, South Africa

Derek Jones, Royal Greenwich Observatory, England

Namir E. Kassim, Naval Research Laboratory, USA

Anne Kinney, Space Telescope Science Institute, USA

Stephen Knapp, Johns Hopkins University, USA

Alexei Y. Kniazev, Special Astrophysical Observatory, Russia

Joachim Krautter, Landessternwarte Heidelberg, Germany 
Martin W. Kümmel, Landessternwarte Heidelberg, Germany

Kip Kuntz, GSFC and University of Maryland, USA

Andrei Kuzmin, Sternberg Astronomical Institute, Russia

Constanze la Dous, Sonneberg Observatory, Germany

Ofer Lahav, Institute of Astronomy, Cambridge, England

Vicki Laidler, Space Telescope Science Institute, USA

Jeff Larsen, University of Minnesota, USA

Barry Lasker, Space Telescope Science Institute, USA

David Leisawitz, Goddard Space Flight Center, USA

Carol Lonsdale, California Institute of Technology, USA

Harvey T. MacGillivray Royal Observatory Edinburgh, Scotland

Steven Majewski, University of Virginia, USA

Robert Mann, Imperial College, England

Michael R.W. Masheder, University of Bristol, England

Peter R. McCullough, Univ of Illinois, Astronomy Dept, USA

Thomas A. McGlynn, NASA Goddard Space Flight Center/USRA, USA

Timothy A. McKay, University of Michigan, USA

Brian McLean, Space Telescope Science Institute, USA

Michael Meakes, Space Telescope Science Institute,, USA

Rene A. Mendez-Bussard, European Southern Observatory, Germany

Francois Mignard, OCA/CERGA, France

Richard S. Miller, Los Alamos National Laboratory, USA

Bruno Milliard, Laboratoire d'Astronomie Spatiale du CNRS, France

Marat Mingaliev, Special Astrophysical Observatory, Russia

Edward C. Moran, IGPP/LLNL, USA

David Morgan, Royal Observatory Edinburgh, Scotland

Jane Morrison, Astronomishes Rechen Institut, Germany

Ulisse Munari, Osservatorio Astronomico di Padova, Italy

Petra Nass, Max-Planck Institut für Extraterrestrische Physik, Germany

Heidi Newberg, Fermilab, USA

Yasushi Ogasaka, NASA/GSFC/JSPS, USA

Tomoharu Oka, The Institute of Physical and Chemical Research, Japan

Sadanori Okamura, Dept. of Astronomy, University of Tokyo, Japan

Seb Oliver, Imperial College of Sci. Tech. and Medicine, England

Patrick S. Osmer, The Ohio State University, USA

Paolo Padovani, Dipartimento di Fisica, Italy

Quentin A. Parker, Anglo-Australian Observatory, Australia

R. Bruce Partridge, Haverford College, USA

Bo Peng, Beijing Astronomical Observatory, China

Eric Perlman, Space Telescope Science Institute, USA

Imants Platais, Yale University, USA

Theodore Pohler, Johns Hopkins University, USA 
Marc Postman, Space Telescope Science Institute, USA Isabella Prandoni, IRA-CNR, Bologna, Italy

Steven Price, Phillips Lab., USA

Luis Ramirez-Castro, Instituto de Astrofisica de Canarias, Spain

Neill Reid, Palomar Observatory, USA

Eric Richards, University of Virginia, USA

Nancy G. Roman, Hughes STX, USA

Diane Roussel-Dupree, ALEXIS Satellite Project-LANL, USA

Stephanie Ruphy, DESPA Observatoire de Paris, France

Delphine Russeil, Observatoire de Marseille, France

Jane Russell, National Science Foundation, USA

Roberto Scaramella, Osservatorio Astronomico di Roma, Italy

Cheryl Schmidt, Space Telescope Science Institute, USA

Marion Schmitz, NED/IPAC/CALTECH, USA

Peter Schneider, Max-Planck-Institut für Astrophysik, Germany

Ethan Schreier, Space Telescope Science Institute, USA

Keith A. Scollick, NASA Goddard Space Flight Center, USA

P. Kenneth Seidelmann, U.S. Naval Observatory, USA

Robin Shelton, NASA/Goddard Space Flight Center, USA

David Shupe, IPAC/Jet Propulsion Laboratory, USA

Richard Smart, Osservatorio Astronomico di Torino, Italy

Robert J. Smith, Institute of Astronomy, Cambridge, England

Konstantin P. Sokolov, Institute of Radio Astronomy, Ukraine

Allesandro Spagna, Osservatorio Astronomico di Torino, Italy

Konstantin Stavrev, Bulgarian Academy of Sciences, Bulgaria

Raphael Steinitz, Ben Gurion University, Israel

Gordon Stewart, Leicester University, England

Conrad Sturch, Space Telescope Science Institute, USA

Alex Szalay, Johns Hopkins University, USA

Larry G. Taff, NASA Headquarters/JPL, USA

Eduard Thommes, Max-Planck-Institut für Astronomie, Germany

Gregory Topasna, Physics Dept., Virginia Tech, USA

Virginia Trimble, University of Maryland, USA

Joachim Truemper, MPI fuer Extraterrestrische Physik, Germany

Sergei Trushkin, Special Astrophysical Observatory, Russia

Milcho Tsvetkov, Bulgarian Academy of Sciences, Bulgaria

Sean E. Urban, US Naval Observatory, USA

Meg Urry, Space Telescope Science Institute, USA

Azita Valinia, NASA/Goddard Space Flight Center, USA

Piet van der Kruit, Rijksuniversiteit Groningen, Holland

Frank Varosi, Hughes STX Corp, USA

Giampaolo Vettolani, Instituto di Radiastronomia CNR, Italy 
Natarajan Visvanathan, MSSSO, Australia

Michael Vogeley, Space Telescope Science Institute, USA

Stefan J. Wagner, Landessternwarte Heidelberg, Germany

Alistair Walker, Cerro Tololo Inter-American Observatory, Chile Jasper V. Wall, Royal Greenwich Observatory, England

William F. Wall, Instituto Nacional de Astrofisica, Mexico

William H. Waller, Hughes STX and NASA/GSFC, USA

Masaru Watanabe, Univ of Tokyo, School of Science, Japan

Gart Westerhout, Baltimore, USA

Nicholas E. White, NASA/Goddard Space Flight Center, USA

Richard L. White, Space Telescope Science Institute, USA

Gerard Williger, NASA/Goddard Space Flight Center, USA

David Windridge, Univ of Bristol - Goldney Hall, England

David Wolfe, Space Telescope Science Institute, USA

Marion I. Zacharias, USRA/USNO, USA

Norbert Zacharias, USRA/USNO, USA

Yongheng Zhao, Beijing Astronomical Observatory, China

Elena Zucca, Osservatorio Astronomico di Bologna, Italy 